\title{
Thermal and Microestructural Characterization of Epoxy-Infiltrated Hydroxyapatite Composite
}

\author{
Pedro Barrionuevo Roese $^{\text {a*, }}$, Sandro Campos Amico ${ }^{\mathrm{b}}$, Wilson Kindlein Júnior ${ }^{\mathrm{a}}$ \\ ${ }^{\mathrm{a}}$ LDSM - Universidade Federal do Rio Grande do Sul \\ 90035-190 Porto Alegre - RS, Brazil \\ ' $L A P O L / P P G E M$ - Universidade Federal do Rio Grande do Sul, \\ 91501-970 Porto Alegre - RS, Brazil
}

Received: December 18, 2008; Revised: February 17, 2009

\begin{abstract}
In this work, hydroxyapatite (HAp) obtained from the deorganification of bovine bones using hot $\mathrm{NaOH}$ solution was used to synthesize a HAp/epoxy infiltrated composite. Infiltration was carried out by vacuum assisted immersion in hot epoxy resin. The resulting composite was characterized regarding polymer content, morphological aspects and flexural strength. The infiltration method used resulted in thorough infiltration of the HAp but some residual porosity remained. Although the epoxy resin showed good interaction with the HAp, high polymer content was achieved and the flexural strength of the composite was higher than that of the original resin or the HAp, composite strength was lower than that of the human cortical bone.
\end{abstract}

Keywords: composite, microstructure, mechanical properties, glass transition

\section{Introduction}

Hydroxyapatite (HAp) is a ceramic material with superior biocompatibility and osteoconductivity properties because of its similarity to the mineral phase of bones ${ }^{1-3}$. Besides its biocompatibility, HAp also shows the ability to promote bone growth and to integrate with surrounding bone tissues playing the role of support and filler of bones ${ }^{3}$, making it a very promising source of biomaterials.

Hydroxyapatite for the synthesis of new biomaterials may come from different sources although synthetic routes are commonly used. However, it is also possible to use HAp from bone, which is obtained by processing bovine or pig bones ${ }^{4}$ resulting in a porous and brittle material. The characteristics of HAp from processed bone and synthetic HAp were studied ${ }^{2}$ and it has been reported that synthetic HAp, despite its chemical similarity to the bone mineral, shows significant differences in crystal morphology compared to bone and HAp obtained from processed bone.

The limitations of using HAp for the synthesis of biomaterials reside in its mechanical properties. For surgery and orthopedic applications, good mechanical properties are mandatory and HAp has very low toughness ${ }^{5-7}$. The most common approach to toughen HAp is to use polymers as a second phase to obtain a HAp-based composite material with mechanical properties comparable to those of bone.

There are two main approaches for obtaining HAp-polymer composites. The most common is to use HAp in particulate form dispersed in a polymer matrix ${ }^{8-10}$. The usual methods to obtain such composites are melt blending and slurry (solution), each of them yielding a different set of mechanical properties.

Typical polymers for slurry and melt blending are PCL (poly $\varepsilon$-caprolactone), PLA (poly L-lactic acid) and UHMWPE (ultra high molecular weight polyethylene). For melt blended HAp-PCL, a tensile strength of $18 \mathrm{MPa}$ was reported ${ }^{11}$, whereas melt blended HAp UHMWPE, reached $28 \mathrm{MPa}^{10}$. On the other hand, $130 \mathrm{MPa}$ flexural strength was reported for hot pressed PLA-HAp slurry ${ }^{8}$. A disadvantage of the slurry method is the need to use an organic solvent for the solubilization of the polymer. Superior mechanical properties obtained with the slurry method come from the improved contact of the polymer matrix with the particles, which is possible due to the low viscosity of the solution in comparison with the polymer melt.

An alternative method to obtain composites with good reinforcement-matrix interaction is the infiltration of a monomer into HAp with interconnected porosity followed by monomer polymerization ${ }^{5,6}$. This method takes advantage of the low viscosity of the monomer and yields improved mechanical properties. These composites are referred to as infiltration composites and a PCL infiltrated HAp achieved toughness comparable to that of human cortical bone ${ }^{12}$.

The porous HAp for infiltration composites may be obtained from two routes: press sintering of HAp particles or foaming ${ }^{3}$. The latter yelds macroporosity whereas the former results in microporosity. In the case of HAp from processed bovine bone, natural porosity results from the deorganification process, but there is a current lack of reported research work addressing infiltration composites with HAp. Thus, the aim of the present work was to obtain and characterize an epoxy infiltrated HAp composite taking advantage of the natural porosity of the HAp, in an attempt to approach the flexural strength of a human bone, which ranges from 60 to $130 \mathrm{MPa}^{8}$.

\section{Materials and Methods}

Hydroxyapatite was obtained from pieces of bovine cortical bone $(101 \times 22 \times 4.5 \mathrm{~mm})$. Deorganification process was carried out based on the procedure described elsewhere ${ }^{13}$. The bones were boiled in a $\mathrm{NaOH}$ solution (4 mol/L) for 24 hours. Then, a new batch of $\mathrm{NaOH}$ solution was used and boiling was carried out for other 24 hours. The HAp was then washed with distilled water until neutral $\mathrm{pH}$ and dried at $100{ }^{\circ} \mathrm{C}$ for 24 hours.

A monoglycidyl ether-based epoxy resin (LY1316) and Epodur 231 curing agent from Huntsman, with a 100:27.5 ratio (wt:wt), were used. Viscosity measurements indicated $60{ }^{\circ} \mathrm{C}$ to be the optimum processing temperature, minimizing viscosity without compromising gel time.

To prepare the composites, pieces of HAp were mounted on a support frame, which kept them apart, and placed inside an acrylic mould. The epoxy and the hardner were mixed, heated to $60{ }^{\circ} \mathrm{C}$, then poured into the mould, allowing full immersion of all HAp pieces. 
The mould was then placed inside a vacuum chamber and vacuum was applied until the evolution of bubbles ceased. Lastly, the pieces were removed, wiped with towel paper to remove excess resin and left to cure overnight. This method is similar to that used to produce ceramic-polymer composites ${ }^{14}$. A disadvantage of such method is that the volume fraction of each component is unknown and needs to be determinated. For comparison, casted samples of pure epoxy resin were molded on the same dimensions of the HAp pieces and following the same heating and vacuum conditions.

Cross-sections of natural bone and HAp were examined with optical (BX51M Olympus ${ }^{\circledR}$ ) and scanning electron (JSM 6060 - Jeol) microscopes. Samples for optical microscopy were ground with sandpaper up to grit \#2000 and then polished using 1- $\mu$ m diamond paste. For scanning electron microscopy, fractured samples were mounted on aluminum stubs and gold sputtered prior to observation. Chemical etching was not necessary.

The specific surface area of the HAp was determined by gas adsorption in a Quantachrome Autosorb Automated Gas Sorption System and calculated according to BET (Brunauer-Emmett-Teller) theory. Density and apparent porosity of the samples were determined based on the Archimedes principle. Thermogravimetric analysis was carried out in a Mettler Toledo TGA/SDTA 851e Thermoanalyser at $20{ }^{\circ} \mathrm{C} / \mathrm{min}$ under oxidizing atmosphere.

Dynamic mechanical analysis of the samples $(18.3 \times 16 \times 2.3 \mathrm{~mm})$ was performed in a TA instruments DMA 2980 with the single cantilever clamp, at $5{ }^{\circ} \mathrm{C} / \mathrm{min}$ heating rate, $1 \mathrm{~Hz}$ frequency and $10 \mu \mathrm{m}$ amplitude. Four-point flexural testing was carried out in an $1105 \mathrm{C}$ Applied Test Systems universal testing machine. The upper and lower spans used were 25 and $50 \mathrm{~mm}$, respectively, and five samples (dimensions: $101 \times 22 \times 4.5 \mathrm{~mm}$ ) were tested at $5 \mathrm{~mm} / \mathrm{min}$.

\section{Results and Discussion}

To verify the removal of organic matter, optical micrographs (Figure 1) and TGA (Figure 2) of the HAp were compared to those of the original bone. In the micrographs, it is possible to see a typical bone morphology (Figure 1a) characterized by the Haversian canal and a circular pattern around it caused by the orientation of the collagen, which constitutes most of the organic matter of bone ${ }^{15}$. Figure $1 \mathrm{a}$ also shows the presence of two resorption cavities, found in most micrographs. In the HAp (Figure 1b), this pattern disappears, leaving

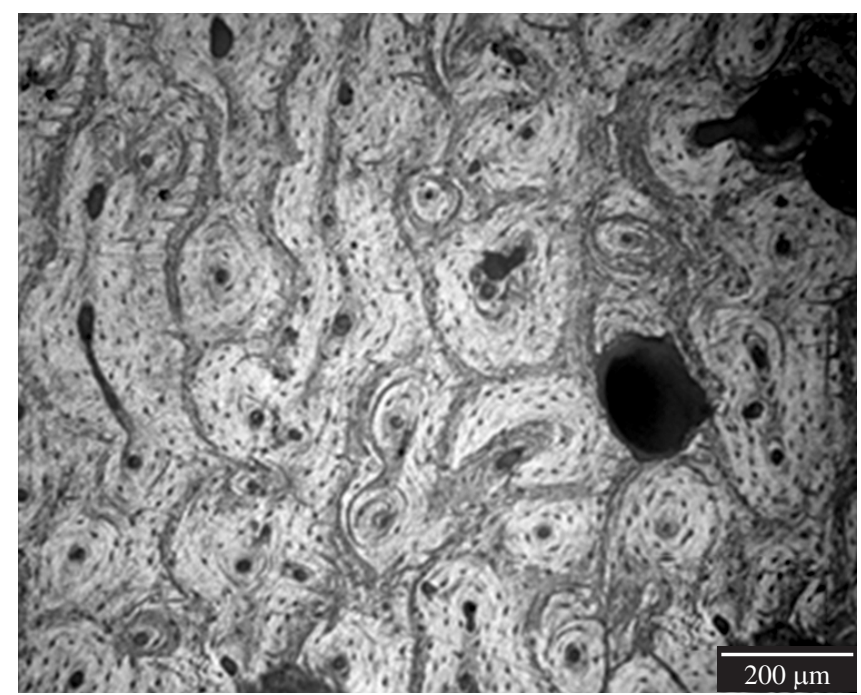

(a) only the Haversian canals. Thus, it may be concluded that most of the collagen was removed.

In the TGA curve of the natural bone, there are three weight loss events: from $30-160{ }^{\circ} \mathrm{C}$, related to the evaporation of water associated with organic matter, between $200-500{ }^{\circ} \mathrm{C}$, attributed to the oxidation of organic matter, and $700-900{ }^{\circ} \mathrm{C}$, attributed to the $\mathrm{CO}_{3}^{-2}$ decomposition $^{13}$. On the other hand, HAp shows only two major events, attributed to the loss of water adsorbed on the HAp surface ${ }^{13}$ and the $\mathrm{CO}_{3}^{-2}$ decomposition. Therefore, the obtained HAp, lacking organic matter, may be considered a suitable ceramic reinforcement for the composite synthesis.

With the scanning electron microscopy (Figure 3), it is possible to verify that the HAp presents the pores, which were part of the Haversian system of the original bone, and also an intricate microporosity, which explains its high specific surface area $\left(91 \mathrm{~m}^{2} \cdot \mathrm{g}^{-1}\right)$. In Figure $3 b$, it is also possible to see micro-cracks in the HAp that appeared as a consequence of the deorganification process.

The experimentally determined density of the composite $\left(1.90 \pm 0.03 \mathrm{~g} . \mathrm{cm}^{-3}\right)$ was intermediate to that of the epoxy resin $\left(1.16 \pm 0.03 \mathrm{~g} . \mathrm{cm}^{-3}\right)$ and of the HAp $\left.(3.16 \mathrm{g.cm})^{-3}\right)^{14}$, resulting in an apparent porosity of $(3.3 \% \pm 1.4 \%)$, which is much lower than that of

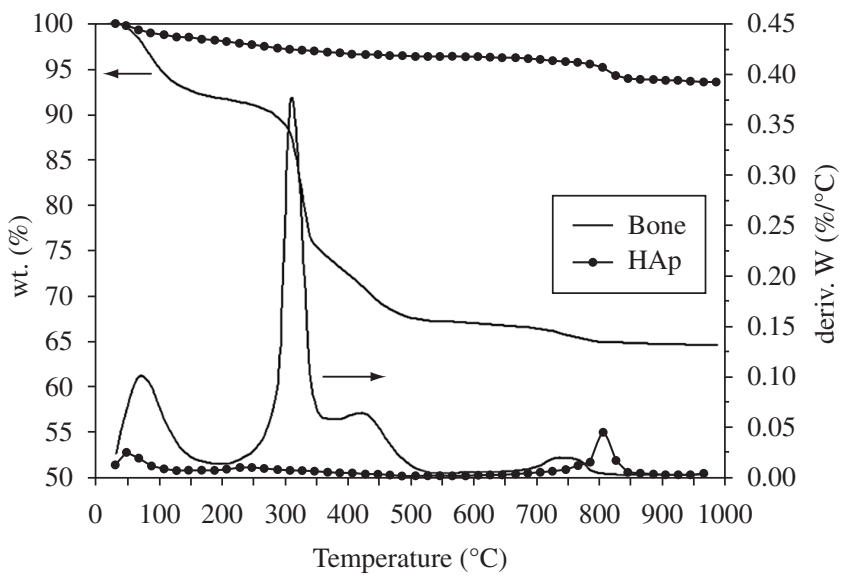

Figure 2. Thermogravimetric curves of bone and HAp samples.

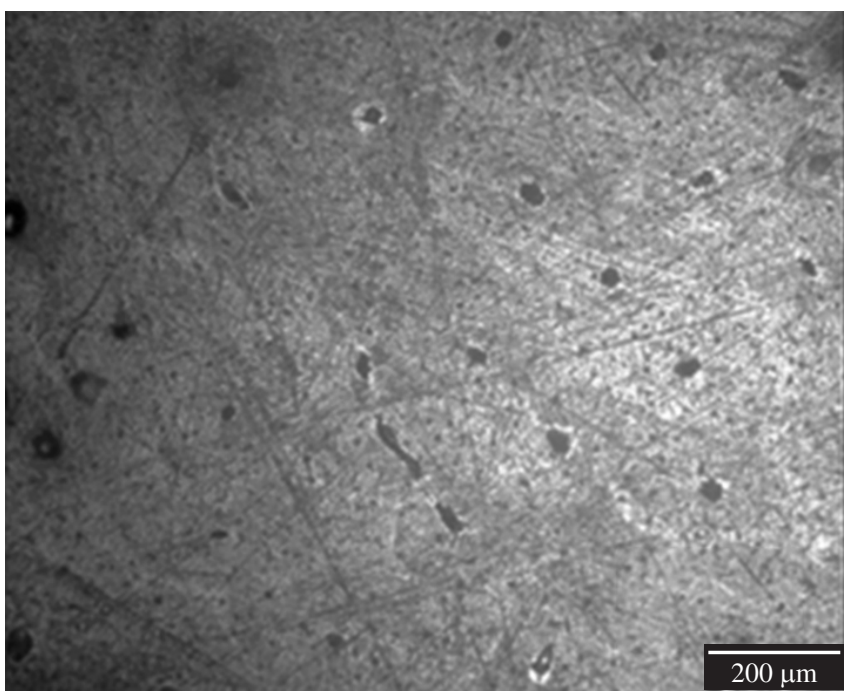

(b)

Figure 1. Optical micrographs: a) Bone and b) HAp. 
the original HAp, $(55.4 \% \pm 1.4 \%)$. Thus, the infiltration process used was able to yield an epoxy infiltrated HAp composite structure.

From the optical micrographs of Figure 4, one can see a radial pattern around the Haversian canals, indicating that those structures were infiltrated first and then the infiltration proceeded radially toward inner regions. Besides, the presence of darker and clearer regions suggests that complete infiltration was not achieved. The presence of residual porosity corroborates the apparent porosity results.

The possible reasons for the incomplete impregnation may be that the viscosity of the resin was too high or the use of vacuum was not fully successful, retaining air bubbles inside the Haversian canals, which, in turn, led to partial filling. Figure 5 shows SEM images of different regions of the composite. In Figure 5a, one can see a region of incomplete infiltration of the Haversian system at the bottom right. Figure $5 \mathrm{~b}$ shows that, despite displaying incomplete infiltration in some regions, the epoxy resin was capable of filling the HAp porous structure.

Figure 6 shows TGA curves of the composite samples. Comparing these curves with the HAp curve in Figure 2, a particularly large weight loss event may be seen between $180-540^{\circ} \mathrm{C}$, attributed to the degradation of the epoxy resin. This weight loss was used to estimate the polymer content in the composite, which was found to be in the $33.9-36.1 \%$ (weight basis) range, or $29.2-31.12 \%$ in volume, i.e. the infiltration method used was able to incorporate a considerable amount of resin into the HAp porous structure. Combining TGA with the density results, the theoretical density of the composite was calculated as $2.05 \mathrm{~g} . \mathrm{cm}^{-3}$. Considering that the composite density was $1.90 \mathrm{~g} . \mathrm{cm}^{-3}$ and that the apparent porosity was $3.3 \%, 92 \%$ of the theoretical density was reached and approximately $4.7 \%$ of closed porosity was present.

The DMA analysis (Figure 7) showed the composite to be much stiffer than the epoxy resin. Besides, for the composite, the glass transition spreads over a wider temperature range, with a higher glass transition temperature $\left(\mathrm{T}_{\mathrm{g}}\right)$. This indicates a good matrix-reinforcement interaction in the composite ${ }^{16}$, reducing interface segment mobility and increasing $\mathrm{T}_{\mathrm{g}}$, contributing for the observed mechanical properties.

Regarding flexural testing, the HAp fractured with just the pretensioning load of $2.5 \mathrm{~N}$ due to its high brittleness, which may be partially attributed to micro-cracks in the HAp pieces. The flexural

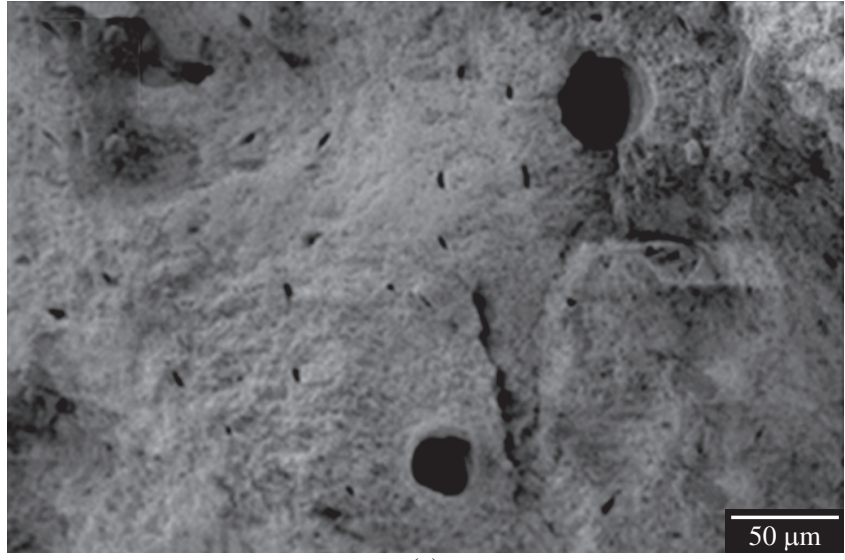

(a)

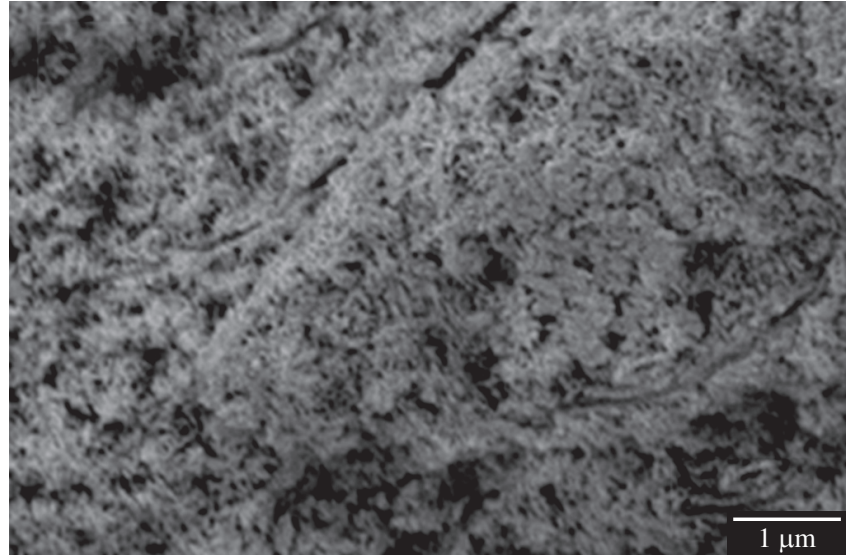

(b)

Figure 3. Scanning electron micrographs of HAp detailing the a) Haversian system and b) micro-pores.

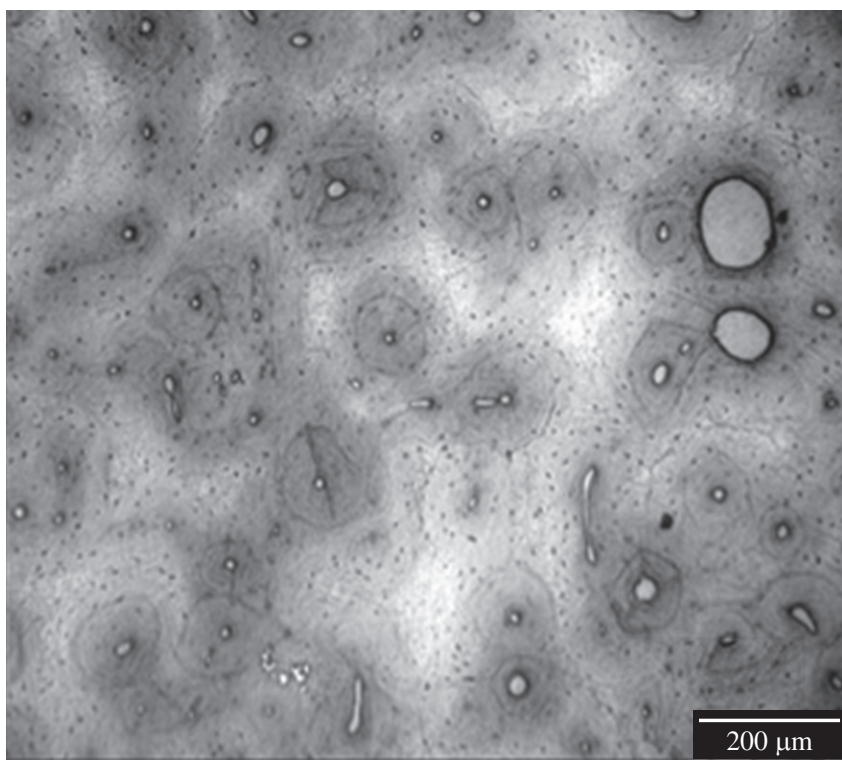

(a)

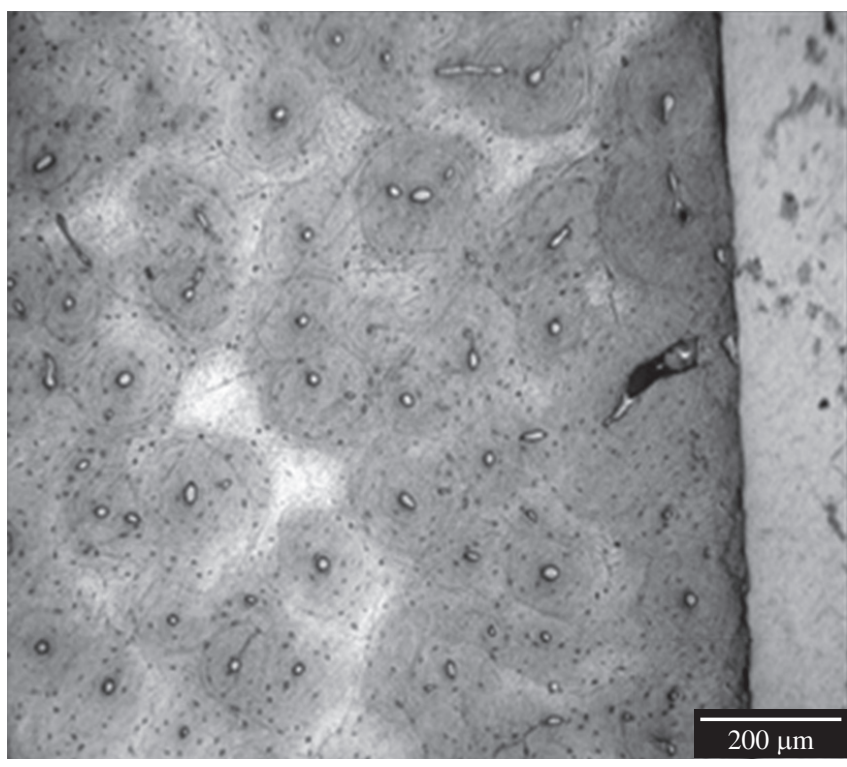

(b)

Figure 4. Optical micrographs: a) Composite center, and b) composite surface. 
strength of the composite reached $26.2 \pm 2.7 \mathrm{MPa}$, which is higher than that of the pure resin, 17.6 MPa, but still below the $60 \mathrm{MPa}$ lower limit of human cortical bone. The literature lacks similar research works for comparison, but PLA-HAp composite obtained by solution techniques reached 100 to $130 \mathrm{MPa}^{8}$. The lower strength observed in the composite produced may be attributed to a few factors: the resin chosen - epoxy, which is not as tough as PCL, the presence of residual porosity in the composite, and the presence of cracks in the HAp.

\section{Conclusions}

Hydroxyapatite was obtained from bones via deorganification in hot $\mathrm{NaOH}$ solution. Most of their organic matter was removed, but the process originated micro-cracks in the pieces, yielding a very brittle material with high porosity, which was used to obtain an infiltration composite with epoxy resin.

Morphological analysis of the composite suggested that the infiltration began at the Haversian canal, proceeding then radially toward inner regions. The polymer content in the composite reached $36 \%$ in weight, $92 \%$ of the theoretical density. Good interaction between resin and hydroxyapatite was found, increasing the glass transition temperature of the epoxy.

The composite presented flexural strength superior to the original materials, but still low in comparison to natural bone and other

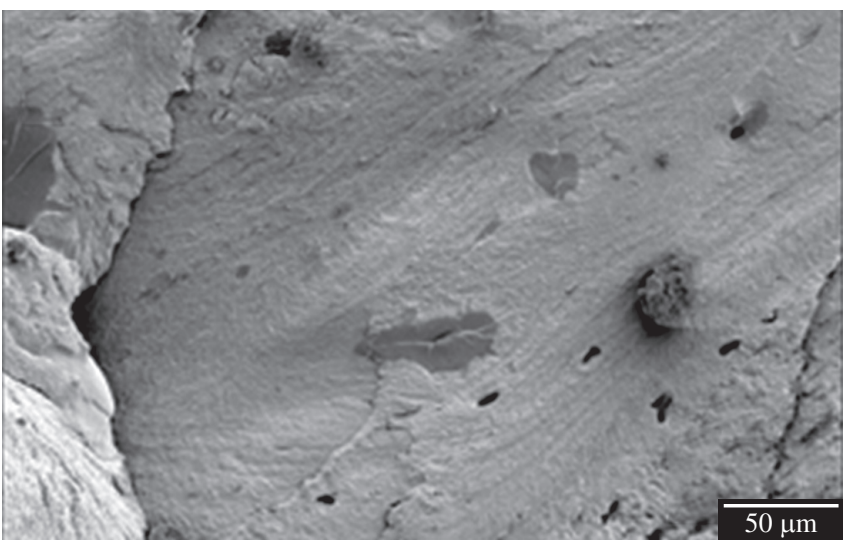

(a)

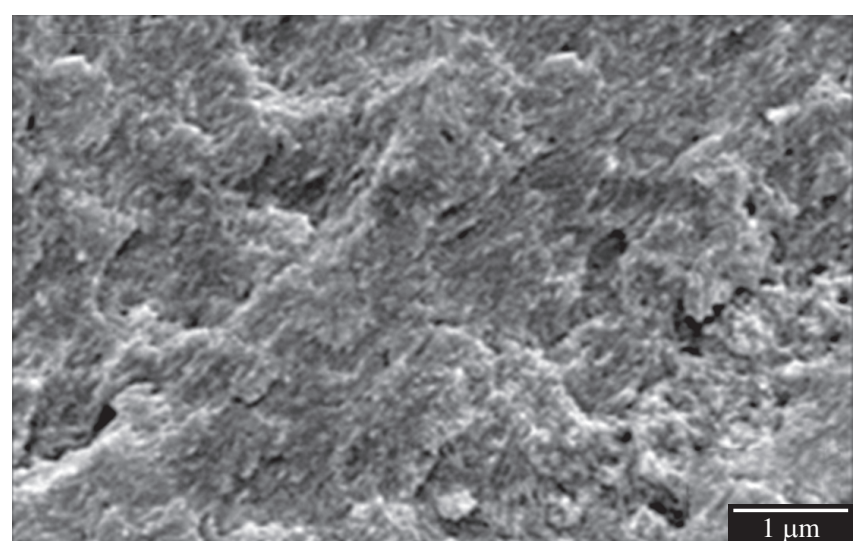

(b)

Figure 5. Residual porosity in a) a Haversian canal and b) in its surroundings.

hydroxyapatite infiltration composites. This was attributed to the limited toughness of the epoxy resin chosen as matrix, the residual porosity due to incomplete infiltration and the presence of microcracks in the HAp pieces.

\section{References}

1. Kalita SJ, Bhardwaj A, Bhatt HA. Nanocrystalline calcium phosphate ceramics in biomedical engineering. Materials Science and Engineering- $C$. 2007; 27(3):441-449.

2. Rosena VB, Hobbsa LW, Spectorb M. The ultrastructure of anorganic bovine bone and selected synthetic hyroxyapatites used as bone graft substitute materials Biomaterials. Biomaterials. 2002, 23(3):921-928.

3. Huang X, Miao XG. Novel porous hydroxyapatite prepared by combining $\mathrm{H}_{2} \mathrm{O}_{2}$ foaming with PU sponge and modified with PLGA and bioactive glass. Journal Biomaterials Applications. 2007; 21(4):351-374.

4. Chlopek J, Rosol P, Morawska-Chochol A. Durability of polymerceramics composite implants determined in creep tests. Composites Science and Technology. 2006; 66(11-12):1615-1622.

5. Nakahira A, Tamai M, Miki S, Pezzotti G. Fracture behavior and biocompatibility evaluation of nylon-infiltrated porous hydroxyapatite. Journal of Materials Science. 2002; 37(20):4425-4430.

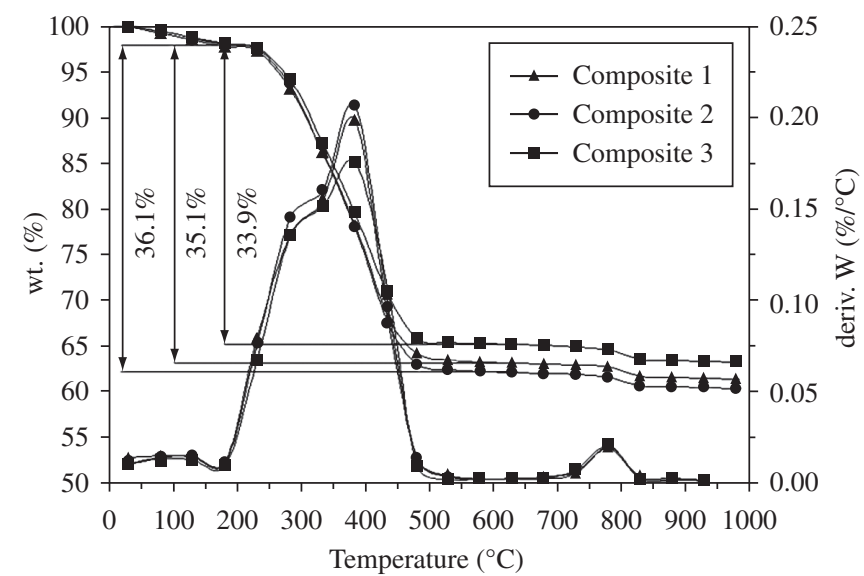

Figure 6. Thermogravimetric curves for three composite samples.

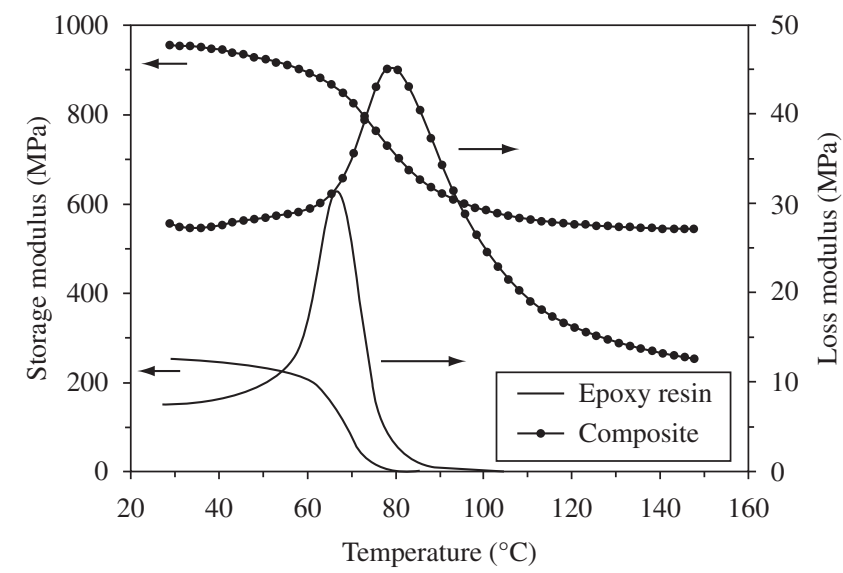

Figure 7. DMA curves for the neat epoxy resin and the infiltration composite produced. 
6. Lien SM, Chien $\mathrm{CH}$, Huang TJ. A novel osteochondral scaffold of ceramic-gelatin assembly for articular cartilage repair. Materials Science and Engineering C. 2009; 29(1):315-321.

7. Zhang $\mathrm{P}$, Hong $\mathrm{Z}$, Yu $\mathrm{T}$, Chen $\mathrm{X}$, Jing $\mathrm{X}$. In vivo mineralization and osteogenesis of nanocomposite scaffold of poly(lactide-co-glycolide) and hydroxyapatite surface-grafted with poly(L-lactide). Biomaterials. 2009; 30(1):58-70.

8. Russias J, Saiz E, Nalla RK, Gryn K, Ritchie RO, Tomsia AP. Fabrication and mechanical properties of PLA/HA composites: a study of in vitro degradation. Materials Science and Engineering C. 2006; 26(8):1289-1295.

9. Jun-Liang Z, Tao F, Yong H, Ke-Wei X. Reinforcing hydroxyapatite/ thermosetting epoxy composite with 3-D carbon fiber fabric through RTM processing. Materials Letters. 2003; 58(1-2):163-168.

10. Liming F, Yang L, Ping G. Processing and mechanical properties of HA/ UHMWPE nanocomposites. Biomaterials. 2006; 27(20):3701-3707.
11. Biqiong $\mathrm{C}$, Kang S. Mechanical and dynamic viscoelastic properties of hydroxyapatite reinforced poly ( $\varepsilon$-caprolactone). Polymer Testing. 2005 ; 24(8):978-982.

12. Pezzoti G, Asmus SMF, Ferroni LP, Miki S. In situ polimerization into porous ceramics: a novel route to tough biomimetic materials. Journal of Materials Science: Materials in Medicine. 2002; 13(8):783-787.

13. Haberko K, Bucko MM, Brzezinska-Miecznik J, Haberko M, Mozgawa W, Panz T, Pyda A, Zarebski J. Natural hydroxyapatite: its behaviour during heat treatment. Journal of European Ceramic Society. 2006; 26(4-5):537-542.

14. Konopka K, Boczkowska A, Batorski K, Szafran M, Kurzydlowski KJ. Microstructure and properties of novel ceramic-polymer composites. Materials Letters. 2004; 58(30):3857-3862.

15. Nyman JS, Reyes M, Wang XD. Effect of ultrastructural changes on the toughness of bone. Micron. 2005; 36(7-8):566-582.

16. Park SJ, Jin FL, Lee CJ. Preparation and physical properties of hollow glass microspheres-reinforced epoxy matrix resins. Materials Science and Engineering A. 2005; 42(1-2):335-340. 